\title{
Bioengineering Models of Cell Signaling
}

\author{
Anand R. Asthagiri ${ }^{1}$ and Douglas A. Lauffenburger ${ }^{1,2}$ \\ ${ }^{1}$ Department of Chemical Engineering and ${ }^{2}$ Division of Bioengineering and \\ Environmental Health, Massachusetts Institute of Technology, Cambridge, \\ Massachusetts; e-mail: asthagir@mit.edu,lauffen@mit.edu
}

Key Words biochemical circuits, biomolecular networks, biophysical interactions, mechanotransduction

- Abstract Strategies for rationally manipulating cell behavior in cell-based technologies and molecular therapeutics and understanding effects of environmental agents on physiological systems may be derived from a mechanistic understanding of underlying signaling mechanisms that regulate cell functions. Three crucial attributes of signal transduction necessitate modeling approaches for analyzing these systems: an ever-expanding plethora of signaling molecules and interactions, a highly interconnected biochemical scheme, and concurrent biophysical regulation. Because signal flow is tightly regulated with positive and negative feedbacks and is bidirectional with commands traveling both from outside-in and inside-out, dynamic models that couple biophysical and biochemical elements are required to consider information processing both during transient and steady-state conditions. Unique mathematical frameworks will be needed to obtain an integrated perspective on these complex systems, which operate over wide length and time scales. These may involve a two-level hierarchical approach wherein the overall signaling network is modeled in terms of effective "circuit" or "algorithm" modules, and then each module is correspondingly modeled with more detailed incorporation of its actual underlying biochemical/biophysical molecular interactions.

\section{CONTENTS}

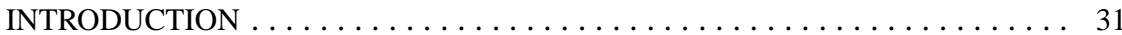

COMPLEXITY IN SIGNAL TRANSDUCTION MECHANISMS $\ldots \ldots \ldots \ldots .33$

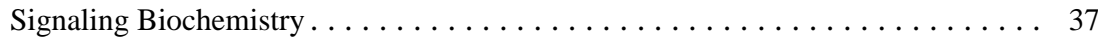

Biophysical Aspects . ............................. 41

TOWARD INTEGRATED NETWORK MODELS $\ldots \ldots \ldots \ldots \ldots \ldots \ldots \ldots$

CONCLUDING REMARKS $\ldots \ldots \ldots \ldots \ldots \ldots \ldots \ldots \ldots \ldots$

\section{INTRODUCTION}

Complex biological processes such as development, tissue function, immune response, and wound healing are orchestrated through precise and dynamic regulation of cell behavior, primarily achieved by active dialogue between cells and their 
environment. This exchange of information, or signals, collectively termed cellular signaling, is based on the cell's ability to "read" environmental cues, "translate" them into intracellular commands, and "react" with appropriate responses. Importantly, to enable truly dynamic regulation, a fourth component-the cell's ability to "write" its own messages into the extracellular space-ensures bidirectional communication.

Consistent with its ubiquity in governing biological processes, the molecular mechanisms underlying a variety of pathologies can be traced to aberrations in one or more of these four aspects of cellular signaling. Autoimmune diseases or graft rejections result from erroneous or undesired recognition (read) of self versus foreign major histocompatibility complexes $(1,2)$. In the autoimmune disease type I diabetes, targeted destruction of pancreatic $\beta$ cells eliminates the production (write) of insulin - a signal carrier that triggers the uptake of glucose by extrapancreatic cells (3). Conversely, the failure of extrapancreatic cells to recognize and translate the presence of an insulin signal leads to type II diabetes (3). Finally, in cancer development, mutations effectively hardwire intracellular signal translation machinery into a promitogenic state even in the absence of mitogenic extracellular cues and induce uncontrolled proliferation, eventually leading to tumor formation (4).

Because of their functional significance in both normal and pathological conditions, cellular signaling molecules provide powerful targets for disease therapy (5). In addition to targeting intracellular signaling components with small molecule inhibitors, protein therapeutics such as soluble antibodies or cytokines may be designed to antagonize or stimulate existing stimulus recognition molecules. In these and other applications such as tissue engineering and biomaterials design, the goal is often to achieve tunable control over cell behavior.

Exploiting this powerful cellular signaling machinery on a rational basis in these applications requires quantitative and mechanistic understanding of how signals govern cell responses. Involvement of numerous species and interactions that collectively propagate signals through an interconnected physiochemical network constrains intuitive predictions of signaling performance. Therefore, growing emphasis has been placed on developing mathematical models to assess the behavior of these complex signaling systems. Moreover, because ongoing characterization of signaling components and pathways is an area of intense research, concurrent progress in inferring the performance of these under-defined systems requires engineering analysis and synthesis. A combination of quantitative experimental approaches, rigorous analysis, and mathematical modeling will be required to tackle the multiple layers of complexity in signal transduction.

The purpose of this review is to highlight conceptually the attributes of signaling systems that necessitate a modeling approach to studying these systems. In conjunction, some previous models are presented to delineate how this approach has provided quantitative insight into regulatory mechanisms governing information flow, including not only biochemical regulation but also biophysical aspects. Given the breadth of this review, we maintain our focus on conceptually describing salient features of cell signaling and on offering our perspective on future directions 
in this rapidly evolving field. We place lesser emphasis on detailed presentation of each element of signal transduction and on all-inclusive coverage of models for signaling, both of which are well beyond the scope of a single review.

\section{COMPLEXITY IN SIGNAL TRANSDUCTION MECHANISMS}

The first level of complexity in cellular signaling derives from the large number of molecules and multiple types of interactions between them. Considering the read functionality alone, detecting extracellular cues requires several classes of sensorstimulus interactions, typically involving receptor-ligand binding (Table 1). While some receptors, such as those for growth factors and cytokines, are found on the cell surface, intracellular receptors bind to small molecules such as steroids capable of passing through the cell membrane. In addition to these soluble factors, extracellular matrix (ECM) proteins and proteins found on adjacent cell surfaces also regulate cell functions through their interaction with adhesion and cell-cell contact receptors such as integrins and cadherins, respectively.

Regardless of the mode of sensing environmental cues, perception of a stimulus must be translated through intracellular signaling mechanisms to affect end-point cell functions. This translation occurs through a cascade of events (Figure 1) involving multiple intracellular species including small molecules, lipids, and proteins. Proteins involved in this process typically possess a modular design composed of

TABLE 1 Stimulus-sensor pairs. Stimuli, typically in the form of protein ligands, are recognized by sensors, typically receptors located on the cell membrane. Most stimuli fall into one of four groups: cell-ECM adhesion, cell-cell contact, soluble factors, and mechanical forces

\begin{tabular}{ll}
\hline Stimulus & Sensor \\
\hline Cell-ECM adhesion (92) & \\
ECM proteins & Proteoglycans (e.g. syndecans) (93) \\
ECM proteins & Integrins (94) \\
Cell-cell contact (92) & \\
Cadherins & Cadherins (95) \\
Major histocompatibility complex (MHC) & T cell receptor \\
Carbohydrates (e.g. sialyl Lewis x) & Selectins (96) \\
Soluble factors & \\
Growth factors, cytokines & Growth factor and cytokine receptors \\
Steroids & Intracellular receptors \\
Mechanical forces & Mechanoreceptors (e.g. integrins) \\
\hline
\end{tabular}




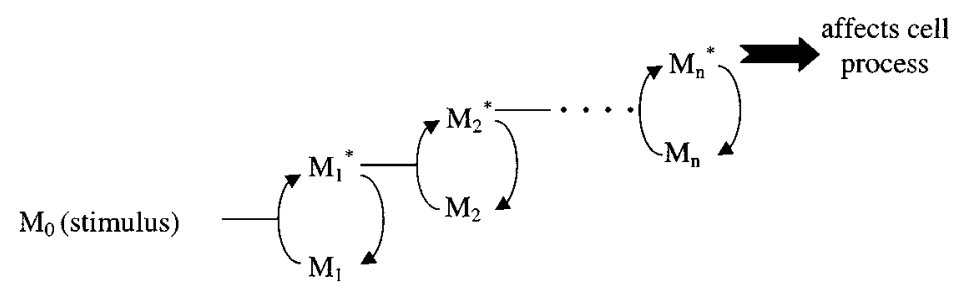

Figure 1 Unidirectional, linear signaling cascade. The simplest view of signal transduction entails a cascade of molecular events initiated by the recognition of a stimulus and culminating in the chemical alteration of an effector molecule $\mathrm{M}_{\mathrm{n}}^{*}$.

different functional domains (Table 2). These domains generally either mediate physical association between a signaling protein and another signaling moiety or catalyze reactions. One pair of common reactions involves the addition (kinase) or removal (phosphatase) of phosphate groups from specific residues on proteins. Enzymatic activity in signaling is not constrained to protein substrates, as kinases and phosphatases target lipid substrates. Other lipid modifications, such as the cleavage of phosphatidylinositol-bis-phosphate (PIP2) to diacylglycerol (DAG) and inositol triphosphate (IP3) by the enzyme phospholipase C (PLC), also occur. Analogously, physical interactions involve the docking of a protein with another protein or lipid. In addition to proteins and lipids, small molecules such as calcium ions play an important role in signal transduction as transporters control their spatial distribution, and enzymatic activity in some cases is dependent on the local concentration of these molecules.

Given all these components, an indication of the immense size of the signaling machinery is offered by the estimate that $\sim 2,000$ and $\sim 1,000$ of the $\sim 75,000$ total genes in the vertebrate genome code for protein kinases and phosphatases, respectively $(6,7)$. These two enzymatic functions comprise just a subset of functional motifs found in signaling proteins, which in turn work with other signaling moieties such as lipids and small molecules.

In addition to the size of the signaling machinery, a second layer of complexityinterconnectivity of signaling biochemistry - is apparent from the fact that signaling proteins often contain multiple functional domains, thus enabling each to interact with numerous downstream targets (Figure 2, species $\mathrm{R}_{1}$ ). One implication of this is that these molecules can serve as branchpoints in signal transduction, as has been observed for proteins such as growth factor receptors (GFR) (8), focal adhesion kinase (FAK) (9), Crk-associated substrate (Cas), Crk, and paxillin $(10,11)$. Some of the pathways initially diverging from this common source (through A, B, and D in Figure 2) may reconverge further downstream (at $\mathrm{E}$ and $\mathrm{G}$ ), revealing that signaling pathways are highly interconnected and that a single stimulus can affect multiple cell functions through this signaling network. Additional network structure is imposed by the fact that different 
TABLE 2 Functional domains within signaling proteins. Signaling proteins typically possess a modular structure composed of different functional domains. These domains either mediate physical interspecies association and binding or enzymatic activity. ${ }^{\mathrm{a}}$ Listed are some common functional groups and corresponding subgroups with unique specificity. Examples of signaling proteins involved in such interactions or enzymes overseeing different substrate modifications are also provided

\begin{tabular}{lll}
\hline Domain functionality & Specificity & Examples \\
\hline Protein-protein interactions & SH2 $\Leftrightarrow$ pY (97) & Grb2-SH2 $\Leftrightarrow$ RTK, FAK \\
& SH3 $\Leftrightarrow$ Pro-rich domain (97) & Grb2-SH3 $\Leftrightarrow$ FAK, Sos \\
& PTB $\Leftrightarrow$ pY (98) & IRS-1 $\Leftrightarrow$ Insulin rec. \\
& WW $\Leftrightarrow$ pS or pT (99) & YAP $\Leftrightarrow$ Yes \\
& PDZ $\Leftrightarrow$ C-term E(S/T)DV (100) & PSD-95 $\Leftrightarrow$ NMDA rec. \\
Protein-lipid interactions (101) & PH $\Leftrightarrow$ phosphoinositides & Akt $\Leftrightarrow$ PI (3, 4) P 2 \\
Kinase activity (7) & Y residue & RTK, FAK \\
& S/T residue & Raf \\
& Y or S/T & MEK \\
Phosphatase activity (7) & Lipids & PI3K \\
& Y residue & SHP-2 (102) \\
& S/T residue & PP2A (103) \\
& Y or S/T residue & MKP-1, Pac-1 (87) \\
Lipotease activity & Lipids & PTEN (104) \\
Lipid modification & Cysteine & Calpain \\
\hline
\end{tabular}

aAbbreviations: DAG, diacylglycerol; FAK, focal adhesion kinase; IP3, inositol triphosphate; PH, plekstrin homology; PIP2, phosphatidylinositol-bis-phosphate; PLC, phospholipase C; Pro-rich, proline-rich; PTB, phosphotyrosine binding; pX, phospho-X residue; rec., receptor; RTK, receptor tyrosine kinase; SH, Src-homology; S, serine; T, threonine; Y, tyrosine.

stimulus-sensor pairs share common signaling machinery to transduce their information. Thus, a single stimulus $\rightarrow$ single pathway $\rightarrow$ single cell function (Figure 1) view of signal transduction must be expanded to a broader perspective: multiple stimuli $\rightarrow$ interconnected signaling network $\rightarrow$ multiple cell functions (Figure 2).

Alongside the magnitude of the signaling machinery and the dynamics of the interconnected biochemistry, biophysical regulation of signal transduction provides a third layer of complexity to these systems. Closer consideration of some of the signaling reactions (ion transporters and protein-protein associations) and molecular components (cytoskeletal proteins, adhesion receptors) suggests that there is a physical aspect to signaling that should not be overlooked (Figure 2). In fact, a 


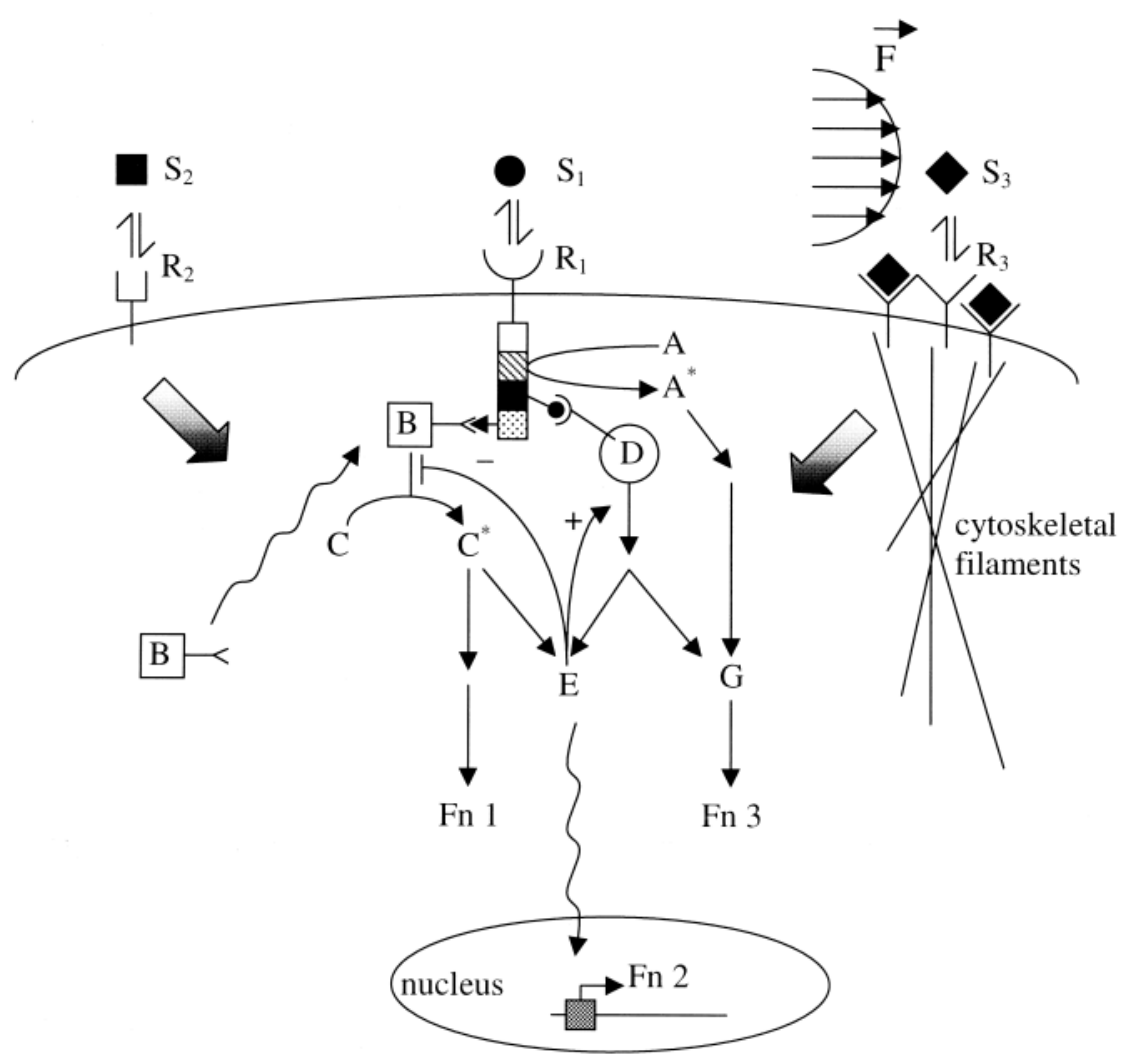

Figure 2 Extension to a more realistic, complex signaling network perspective. A more expansive perspective on signaling considers a network of biochemical and biophysical events that are initiated in response to the detection of multiple chemical stimuli (S) or forces (F) by different sensor receptors $(\mathrm{R})$. This network involves branch points (cytoplasmic domain of $\mathrm{R}_{1}$ ) from which multiple pathways emanate and convergence points $(\mathrm{E}, \mathrm{G})$ where pathways meet to impart coordinated regulation of multiple cell functions (Fn). Also depicted are biophysical regulatory elements such as translocation and localization of proteins (wavy lines) and force redistribution through cytoskeletal filaments.

eukaryotic cell cannot be modeled generally as a "well-mixed bag of molecules" without grossly oversimplifying important aspects of signaling regulation. Rather, eukaryotic cells are constructed with well-defined molecular architecture, with proteins and organelles placed in appropriate locations to optimize function. Because information transfer along a signaling pathway strongly depends on interplay between its biochemical and biophysical aspects, models coupling these two factors are essential in delineating a more complete mechanistic understanding of signal propagation. 


\section{Signaling Biochemistry}

In considering the interconnected biochemistry of signal transduction, an engineering objective would be to determine information flow through these pathways and to redistribute that flow to favor desired cellular responses. Tracking information flow will require identification of multiple metrics, rather than a single universal descriptor, for information content as these networks involve different types of species (e.g. lipids, small ions, proteins) and proteins with multiple functional domains. In the case of signaling proteins, various biochemical properties such as degree of phosphorylation of a protein scaffold or enzymatic activity level of a kinase may serve as information metrics. Moreover, because each signaling protein often possesses multiple functional domains, several such candidate indicators may apply to a single protein. Additionally, the packaging of information may not remain consistent as it traverses the pathway. For example, a pathway may begin with a scaffolding event between a phosphorylated protein $\mathrm{R}_{1}$ and its target protein B (Figure 2). Once this target protein B docks, it may then acquire catalytic activity for its downstream substrate $\mathrm{C}$.

For the special case in which each component in a pathway possesses a single property indicative of its information content, steady-state efficiency of signal transfer from a stimulus $\left(\mathrm{M}_{0}\right)$ to a target process $\left(\mathrm{M}_{\mathrm{n}}\right)$ can be related to the steadystate efficiency of information transfer at each rung of the pathway (Figure 1). Kholodenko et al define a sensitivity factor $\left(\lambda_{\mathrm{i}, \mathrm{j}}\right)$, analogous to a response coefficient in metabolic control analysis (13), for information transfer between an upstream signaling molecule $\mathrm{M}_{\mathrm{i}}$ and a downstream component $\mathrm{M}_{\mathrm{j}}$ as the ratio of fractional change in $\mathrm{M}_{\mathrm{j}}$ activity to the fractional change in $\mathrm{M}_{\mathrm{i}}$ activity in the limit in which the fractional change in $M_{i}$ activity approaches zero (Figure 3) (12). This limit indicates that this ratio is a local sensitivity factor whose value depends on the level of $\mathrm{M}_{\mathrm{i}}$ activity. Local sensitivity ratios greater than one indicate a sensitivity magnification such that a fractional change in $\mathrm{M}_{\mathrm{i}}$ activity is magnified to a larger fractional change in $M_{j}$ activity. Additionally, in the case where $i=0$ and $j=n$, we obtain the stimulus-response local sensitivity factor $\left(\lambda_{0, \mathrm{n}}\right)$, which has been shown to equal the product of the local sensitivity factors at each level of the pathway (i.e. the product of all $\lambda_{i, i+1}$ for $i=0$ to $\left.n-1\right)(12,14)$.

Whereas local sensitivity gives an assessment of how $M_{j}$ activity changes for small perturbations in $\mathrm{M}_{\mathrm{i}}$, its value is a function of specific levels of $\mathrm{M}_{\mathrm{i}}$. A more global measure of sensitivity $\left(\lambda_{i, j}\right)$ can be defined as the fold increase in $M_{i}$ required to elicit a shift from $10 \%$ to $90 \%$ maximal $\mathrm{M}_{\mathrm{j}}$ activity (Figure 3) (15). When $\mathrm{M}_{\mathrm{j}}$ activity is a monotonically increasing, sigmoidal function of $\mathrm{M}_{\mathrm{i}}$ activity, a curve described by the Hill equation would effectively describe the $\mathrm{M}_{\mathrm{j}}$ activity dependence on $\mathrm{M}_{\mathrm{i}}$ activity (16). The Hill coefficient $(\eta)$ parameter from this curve fit relates to global sensitivity by the equation $\lambda_{\mathrm{i}, \mathrm{j}}=(81)^{1 / \eta}(15)$. Importantly, this global sensitivity is not a function of specific $\mathrm{M}_{\mathrm{i}}$ activity levels, but a property of the entire $M_{j}-M_{i}$ dose-response curve. It should be noted that the stimulus-response 


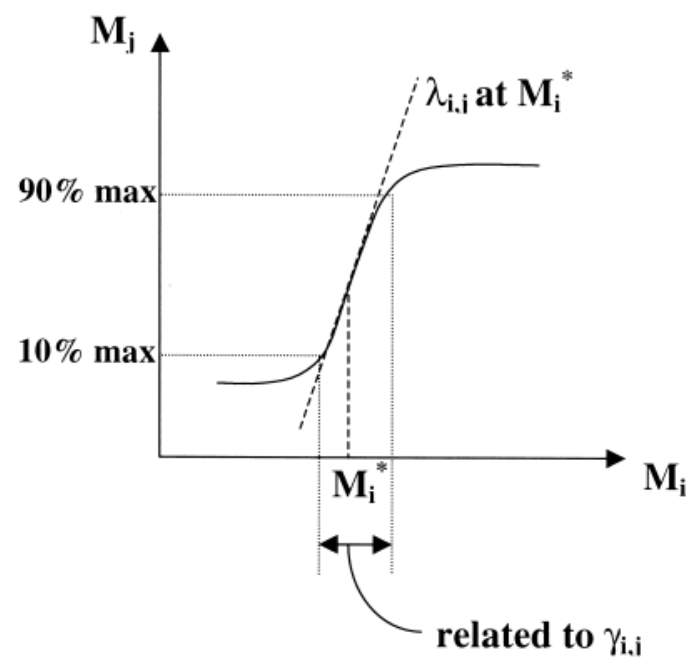

Figure 3 Local and global sensitivity of steady-state signal propagation. In the specific case where information content of signaling events are fully captured by their steady-state values, the slope at specific points along the steady-state functional dependence between two species in a pathway provides a measure of local sensitivity. Also, in the case in which this dependence is a monotonically increasing, sigmoidal function, a global sensitivity measure can be obtained by fitting the curve to the Hill equation.

global sensitivity $\left(\lambda_{0, \mathrm{n}}\right)$ does not adhere to the same property as the stimulusresponse local sensitivity and is not simply the product of global sensitivities at each level of the pathway (17). Responses with high stimulus-response global sensitivity as measured by a Hill coefficient $>1$ are labeled "ultrasensitive" responses because their response elevates from $10 \%$ to $90 \%$ of maximal level over a smaller range of stimulus amount than would a Michaelis-Menten response that possesses a Hill coefficient of 1 . This ultra-sensitive behavior can be derived from different sources: operation of enzymes in a region of saturation with respect to their protein substrates (termed "zero-order" sensitivity) $(15,18)$, the presence of a stoichiometric inhibitor (19), the involvement of the same effector in multiple steps of a pathway (19), and two-collision, distributive mechanisms for dual modification-dependent activation of a signaling component $(18,20)$.

This definition of global sensitivity relies on a monotonic, sigmoidal relationship between stimulus and response. However, other functional relationships are also seen in cases such as biphasic dependence of migration speed on cell-substratum adhesivity (21) or differential transcription of genes resulting in at least three different behavioral states in response to changes in levels of one stimulus or morphogen (22). In fact, in Xenopus blastula cells, morphogen-bound receptor numbers of 0, 100, and 300 commit cells to no, Xbra, or Xgsc transcription, respectively (23). 
Given this transcription dependence on a small number of species, stochastic contributions to gene transcription is likely to yield individual cell response variability. In a stochastic simulation of gene expression in a simple system wherein a protein product encoded by one gene promotes the expression of a second gene, the level of protein transcribed from the second gene was found to increase in "bursts" separated by random time intervals (24). Extending this approach to a complex genetic circuit governing phage $\lambda$ choice of lytic-lysogenic pathway reveals that a stochastic kinetic model accurately predicts pathway choice at different phage:cell ratios (25). Stochastic modeling has also been applied in receptor-ligand mediated detection of chemoattractant gradients and directionality of migration $(26,27)$. Whether cells are situated in the absence or presence of a gradient, their migration can be characterized as a random walk with only the degree of directional bias favoring the gradient in the latter case. Thus, even in the presence of a gradient, stochastic variations in receptor-ligand binding kinetics can result in random error of sampling equivalent in some cases to the $1 \%$ gradient difference that determines cell assessment of a chemical gradient (28). Hence, even in the presence of a gradient, the cell can be randomly oriented in the "wrong" direction despite the random biased motion of cells "moving up" a chemoattractant gradient. Both of these examples demonstrate often-important features of cell signaling responses: $(a)$ a relatively small number components and $(b)$ consequent variability of measured outcome of behavior of individual cells across a population.

As suggested by these stochastic models, kinetic analysis can offer new and significant insight into signaling performance not evident from considering steadystate behavior of signals alone. Rapid positive and negative regulatory mechanisms characteristic of signaling biochemistry often produce signals whose information content resides in their transient features. Broadly, these transient signals can be categorized into cyclical and noncyclical types.

Among the noncyclical category are signals that undergo no, partial, complete, or over adaptation (29) (Figure 4). It is possible that even in scenarios such as no or partial adaptation, the rate of initial signal generation determines downstream response and not the later steady-state level of signal attained. This might be the case if a feedback decoupling step disconnects the signal from its downstream target in a time-scale much shorter than that needed for the signal to reach steadystate. In this scenario, the downstream target only has the opportunity to respond to early levels of signal, and the later steady-state level has no implication for downstream signal propagation.

In complete adaptation, the steady-state level of the signal returns to its initial value following a stimulus challenge, suggesting that the stimulus fails to propagate any new information through this signaling molecule to an end-point cell function. However, transient signals have been shown to regulate not only transient cell responses occurring within the same time-scale as the signal, but also cell functions that occur well after the transient signal returns to its initial level.

An example of the former occurs in the regulation of bacterial chemotaxis. In response to an attractant, peritrichous bacterium Escherichia coli bias their 


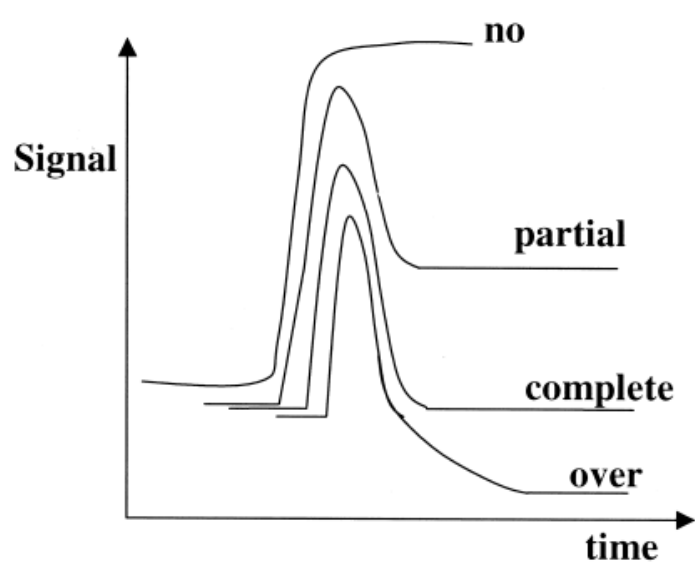

Figure 4 Modes of signal adaptation. Signals are often dynamically regulated such that exposure to stimulus initiates a transient phase after which the signal plateaus at some steadysteady state level. Comparison between steady-state and initial level of signal suggests four forms of adaptation.

random walk by taking fairly straight "runs" while reducing their frequency of "tumbles," allowing the organism to move up an attractant gradient (30). Whereas the default condition is to tumble, when bacteria are exposed to a stimulant such as aspartate a series of fairly well-characterized signaling events leads to an increase in straight runs $(30,31)$. These signals return quickly to basal levels, and the motor is again returned to the default tumble mode (32). This rapid, complete adaptation is preserved over five orders of magnitude of aspartate concentrations and is a feature that permits sensitive response to chemical gradients (33).

In contrast to concurrent complete adaptation of transient signal and response, signals that undergo complete adaptation also affect late cell responses. Transient activation of extracellular signal-regulated kinase 2 (ERK2) in mammalian cells has been shown to coincide with cell proliferation (34) and progression through G1 (35) or S (AR Asthagiri, CA Reinhart, AF Horwitz, DA Lauffenburger, submitted for publication; 37) phase of the cell cycle, depending on the cell system and mode of stimulation. In the case of stimulation of Chinese hamster ovary (CHO) cell by adhesion to fibronectin, ERK2 activation with lifetime as brief as $\sim 15 \mathrm{~min}$ affects DNA synthesis occurring $\sim 15 \mathrm{~h}$ after exposure to stimulus (36). Moreover, the integral of the transient ERK2 activity time-course serves as an effective metric for encapsulating the information content of this signal and permits quantitative correlation to levels of both upstream stimulus (38) and downstream DNA synthesis (36). Because a large number of signaling responses have been shown to have similar pulse-like activation time-courses, these findings extend the important notion that signals undergoing complete adaptation may be equally significant information transducers as those signals that maintain a supra-basal steady-state level.

Another class of transient signals demonstrates cyclical behavior. Oscillations of cytosolic free calcium $\left(\mathrm{Ca}^{2+}\right)$ occur as either baseline spikes or sinusoidal oscillations (39). An increase in agonist amount elevates the frequency without affecting the amplitude of baseline $\mathrm{Ca}^{2+}$ spikes (40). Both positive and negative feedback 
mechanisms are central to creating cyclical signals whose frequency is dependent on agonist dose (39). Model predictions indicate that signal frequency contains the information content of the $\mathrm{Ca}^{2+}$ response as it relates the $\mathrm{Ca}^{2+}$ signal to the level of cell response (41); recent experimental reports support this concept in relation to both cell response (42) and molecular mechanisms (43). The advantage of frequency-encoded signals may be in the detection of low levels of stimulus, as correspondingly low frequency of calcium spikes may permit the build-up of a downstream signal, provided the downstream modification is not reversed within the same time scale as the periodicity of $\mathrm{Ca}^{2+}$ spikes $(39,41,44)$.

In addition to calcium, periodic behavior of cyclins and cyclin-dependent kinases regulates progression through the cell cycle, and unlike $\mathrm{Ca}^{2+}$ pulses that typically occur in short time scales (seconds-minutes) the cell cycle has signaling periodicity on the order of hours. Phase plane analysis shows that kinetic parameters that maintain a noncyclic, supra-basal steady-state value of cyclin-cdk activity lead to cell cycle arrest, and only certain values of kinetic parameters are conducive to oscillations in cyclin-cdk activity, essental for traversal through the cell cycle (45).

Both cyclical and noncyclical transient signals play a crucial role in information transfer, necessitating models that analyze both transient and steady-state aspects of signal transduction. Accordingly, kinetic models must include bi-directional information transfer mechanisms such as positive and negative feedback regulation that impart complex dynamic features to signal transduction. This emphasis on transient behavior contrasts with standard metabolic engineering analysis, which currently emphasizes steady-state flux distribution in metabolic pathways (13). Although comparison between flux of information in signal transduction and flux of metabolites in metabolic pathways is metaphorically descriptive (46), information flux analysis will require a different mathematical framework than that used to follow mass exchange of atomic species from one metabolite to another. This alternative approach is required not only to enable dynamic analysis of information conversion among various biochemical properties of different classes of signaling components, but also to incorporate the unique biophysical regulatory elements prevalent in signal transduction.

\section{Biophysical Aspects}

Closer consideration of signaling components and reactions suggest biophysical regulation of signal transduction adds another layer of complexity to these systems on top of contributions from the large number of signaling components and biochemical interconnectivity. Protein-protein and protein-lipid associations specifically distribute signaling moieties to cellular locations, thereby physically channeling information flow. Often these associations lead to large multi-protein complexes wherein intermolecular presentation and access may affect signal propagation (47). Moreover, physical linkage between intracellular cytoskeletal filaments and ECM provide means for force transmission into the cell, where consequent biochemical signaling events are induced. Because spatial distribution and 
mechanical forces play an important and widespread role in signal transduction, models coupling these biophysical aspects to descriptions of signaling biochemistry are required to obtain a more complete mechanistic understanding of signal propagation.

Spatial Distribution Ensuring proper intracellular and extracellular communication requires that signals not only possess appropriate magnitude and kinetics, but also route to specific locations. In immune responses, cytokines that attract leukocytes and macrophages must be distributed along a "track" that these cells can follow to a site of injury. In development, ligand distribution can determine spatial patterning within tissues by selectively inducing differentiated phenotypes in cells at proper locations. Within the cell, signals for gene expression need to be translocated into the nucleus, where transcription can be initiated, whereas commands for migration may be distributed along the cytoskeleton to induce contraction or at focal adhesions to regulate cell-substratum adhesivity.

Within the extracellular environment, the ECM exercises spatial control by behaving not as a homogenous stagnant hydrogel but as a dynamic space wherein both passive and active transport mechanisms regulate location of extracellular cues. While diffusion of soluble proteins through ECM is retarded owing to viscous drag and tortuosity that result from the presence of protein and polysaccharide scaffolds (48), additional properties of ECM further modulate spatial distribution of soluble ligands. For example, transport of basic fibroblast growth factor (bFGF) through ECM is a function not only of its diffusion in the matrix, but also its binding to heparan sulfate found on proteoglycans in the matrix or on cell surfaces. Blocking bFGF interaction with proteoglycans with addition of soluble heparin allows bFGF to travel with a higher apparent diffusion coefficient (49). The potential significance of proteoglycan-mediated regulation of bFGF transport is depicted in an in vitro study of the ability of bFGF to induce morphological changes in bovine aortic endothelial (BAE) cells. When applied at the center of a monolayer of BAE cells, bFGF plus heparin stimulated morphological changes at a 10-fold greater radius than bFGF alone (50). Hence, modulating proteoglycan-bFGF interaction may provide dynamic spatial control over bFGF-induced responses in tissues.

A different transport mechanism is employed in regulating distribution of the ligand Wingless (Wg) in Drosophila development (51). Diffusion of this Wnt protein is also restricted, perhaps partially by its interaction with proteoglycans; however, in this system, an active vesicular transport mechanism shuttles this ligand over long distances and in a spatially-directed manner (52). A cell-surface receptor, possibly different from the signal-transducing $\mathrm{Wg}$ receptor (53), binds and internalizes the ligand, which is consequently released back to the extracellular milieu. By polarizing the internalization and release along the anterior-posterior axis of each cell, the ligand is effectively directed in a cell-driven and likely energy-consuming mechanism. 
Another cell-mediated control of ligand transport is observed in autocrine cells that secrete ligands for which they also possess receptors (54). First-glance observation may suggest that an autocrine loop is a roundabout method of autonomous regulation of single cell behavior, perhaps more efficiently driven if all commands are conveyed intracellularly. However, implications of autocrine signaling are better appreciated if we consider system conditions that permit escape of ligand signal into surrounding milieu. By modulating parameters such as cell density, the radius over which ligand can communicate its signal can be expanded to encompass a community of cells or constrained to regulate a local group of cells $(55,56)$.

At a single cell level, these properly positioned extracellular cues are typically recognized at the cell membrane, where spatial issues further regulate stimulus sensing and intracellular signal propagation. In some cases, membrane receptors initially propagate their detection of a ligand through a cascade of events involving primarily membrane-associated proteins. For example, ligated seven transmembrane-spanning $\mathrm{G}$ protein-coupled receptors mediate dissociation of GDP-associated trimeric $\mathrm{G}$ proteins into a $\mathrm{G}_{\beta \gamma}$ and GTP-associated $\mathrm{G}_{\alpha}$ subunit, each of which go on to affect the activity level of downstream target enzymes (57). For membrane-constrained reactions operating in a diffusion-limited regime, models of collision-coupling reactions involving enzymatic conversion of substrate to product produce a local zone of substrate depletion if the substrate modification is irreversible (58). This depletion zone arises because of the comparably faster rate of substrate consumption than diffusion of new substrate into the microenvironment of each enzyme. These localized reductions in substrate concentration result in a reduced overall reaction rate compared to the case in which even spatial distribution is maintained. Hence, relative balance of diffusion and reaction rates in combination with signaling biochemistry specifications such as irreversibility of substrate modifications jointly determine spatial distribution of signaling components and rate of signal transfer.

Spatial regulation of information transfer also derives from dynamic allocation of signaling components to different compartments within the cell (59). For example, in GFR-mediated signaling, ligand-bound, autophosphorylated GFR (GFR*) associates with substrates that in turn affect their targets (8). However, progression through this biochemical pathway is regulated by redistribution mechanisms that either maintain GFR* at the plasma membrane or sequester it into small intracellular vesicles (endosomes) that invaginate from the cell membrane. This partitioning of a subpopulation of GFR* and its associated substrate (GFR ${ }^{*}$-substrate) into the endosome may inhibit access to target molecules that localize solely to the plasma membrane. A theoretical model of this compartmentalization effect predicts that signal transfer through GFR* may be modulated even if the biochemical activity of the GFR*-substrate is equal in both compartments (60). Experimental evidence of this compartmentalization effect has been shown in the epidermal growth factor receptor (EGFR) system wherein phosphorylated EGFR (EGFR*) binds and 
activates phospholipase C- $\gamma$ (PLC $\gamma$ ), which in turn cleaves PIP $_{2}$ into $\mathrm{IP}_{3}$ and DAG. Despite equal levels of PLC $\gamma$ activity in cells in which EGFR* was maintained predominantly in the membrane or in endosomes, the latter endosomal sequestration of EGFR* reduced $\mathrm{PIP}_{2}$ hydrolysis, suggesting that access to $\mathrm{PIP}_{2}$ thwarted signal propagation through this pathway (61).

Eventually, the signal that has been sensed and partially processed near the membrane is transferred into the cell interior, often with continued involvement of protein-protein or protein-lipid associations. In addition to involvement in important regulatory functions such as compartmentalization, these interspecies associations concentrate specific cytosolic proteins within close proximity of the information-rich membrane. If this translocation raises the local concentration to saturating levels, signal transfer may occur with "zero-order" ultrasensitivity even though averaged concentrations based on the entire cell volume would not predict this behavior (62).

However, nonspecific localization of signaling molecules to areas of high signaling activity containing numerous signaling moieties may yield undesired cross talk between what should be unrelated pathways. Additionally, different stimuli often use common signaling components to affect varied end-point cell functions, further increasing the possibility of erroneous information exchange between pathways. In some cases, preserving fidelity of stimulus-response connections is achieved through scaffold proteins that bring molecules together and physically wire a specific pathway leading to an appropriate cell response $(63,64)$. In Saccharomyces cerevisiae, the kinase Ste11 is involved in pathways that affect both mating response and cellular osmolarity (63). The choice between these two pathways is determined by which scaffold protein, Ste 5 or Pbs2, preferentially recruits Ste11. Because each scaffold protein is associated with a different set of downstream molecules, differential scaffolding of Ste11 commits it to effect a particular cell response.

In addition to determining the dynamics and fidelity of signaling, spatial effects also target signals to proper locations in the cell at which an end-point activity is performed. Signals affecting migration may target to sites of cell-substratum interaction while those regulating gene expression, cell cycle progression, or differentiation are directed to the nucleus. Some scaffolding proteins, such as A-kinase anchoring proteins (AKAPs), play a prominent role in this aspect of spatial regulation (64). AKAPs possess one domain that localizes them to a particular cellular location while another domain interacts with the regulatory domain of protein kinase A (PKA). A family of $\sim 30$ different AKAPs serve to position PKA to certain subcellular locations such as along actin filaments or microtubules or with calcium channels or NMDA receptors in post-synaptic junctions.

From this overview of spatial distribution effects on signal propagation, it is evident that signaling constituents within a cell do not perform under "well-mixed" conditions but are precisely positioned to optimize function. Importantly, this positional regulation is not established as a static backdrop on which dynamic biochemistry then propagates the signal. Rather, because biochemistry and spatial 
distribution are interconnected with one determining the other, spatial effects must be modeled as dynamic features. This dynamic character of spatial distribution provides an alternative point of regulation of information flux on top of signaling biochemistry specifications.

Mechanical Forces In addition to chemical stimuli such as soluble cytokines and ECM proteins, mechanical forces also induce changes in cell behavior. External load regulates chondrocyte proteoglycan synthesis, thereby altering mechanical resistance of cartilage to compressive stresses (65-67). In vivo mechanical load on cardiac and skeletal muscle determines muscle mass, and in vitro cultured cardiac and skeletal myocytes subject to static stretch undergo hypertrophy—an increase in protein synthesis without DNA synthesis $(66,68-70)$. In endothelial cells lining blood vessel walls, exposure to shear stress changes cell morphology and gene expression $(71,72)$. Also, transmural blood flow through interstitial spaces in tissue imparts shear stress on smooth muscle cells, altering their cell behavioral state (73).

Translating these externally applied forces into cellular responses may partly involve intracellular transmission of forces originally sensed at the cell membrane (74). Mechanoreceptors such as integrin adhesion receptors and cell-cell contact receptors are ideal force conductors, as these receptors bind to both ECM proteins and intracellular cytoskeletal proteins, thereby physically linking the external protein matrix to the internal cell shape-defining scaffold $(74,75)$. Micromanipulation of ligand-coated microbeads attached to cell surfaces through specific integrinligand interactions transmits forces across the membrane through the cytoskeleton and results in nuclear deformations (76). A tensegrity model proposes that forces that deform ECM or ECM-cell contacts result in alterations in the configuration of all three filament systems - actin, intermediate, and microtubules-shifting the internal stress field along these cables from one state to another (74).

Either through this global mechanical deformation and stress redistribution (74) or through force-mediated effects on transmembrane linkages alone (77), a variety of biochemical events are initiated, many of which are similar to those induced by recognition of chemical stimuli. Endothelial cell exposure to shear stress induces intracellular biochemical events with time scales ranging from $<1 \mathrm{~min}$ to $>6 \mathrm{~h}$ (71). The rapid signaling responses include activation of potassium ion channels and changes in intracellular calcium levels, whereas late responses involve cell morphological realignment and gene regulation (71). Exposure to shear stress also induces phosphorylation of growth factor receptors and members of prototypical GFR signaling pathways such as Shc, ERK, and JNK (78). Importantly, blocking these biochemical events abrogates force-induced cellular response. Expression of the SH2 domain of Shc reduces shear stress-induced ERK2 activation in endothelial cells (78), whereas blocking potassium channels inhibits shear-induced TGF- $\beta 1$ gene transcription (79). These findings emphasize the significant intermediary role played by biochemical signaling events in converting mechanical forces into cellular responses. 
Hence, determining mechanisms whereby mechanical forces alter biochemical interactions in signal transduction will provide significant insight into understanding force-mediated regulation of cell behavior. Several potential mechanisms have been proposed. Force-mediated geometrical reconfiguration of the cytoskeleton may reduce interfilament spacing and bring filament-associated proteins within closer proximity of each other, potentially enhancing chemical reactions by elevating local concentrations of interacting species (74). Alternatively, forces distributed along noncovalent protein-protein bonds may alter bond formation and dissociation kinetics (80). This is particularly applicable to cytoskeletal proteins and signaling molecules that form multi-protein complexes at sites of cell-ECM and cell-cell contact. At these junctions, any stress on the surrounding matrix or neighboring cell would be imparted into these multi-protein complexes wherein interprotein bonds would be stressed, thereby altering chemical signal transduction events (74). Finally, forces may affect enzyme functionality by physically altering molecular structure (81). This would be equivalent to a mechanical version of allosteric regulation analogous to the well-known chemically induced alteration in enzyme conformation. Some ion channels have been shown to interact with the cytoskeleton and may be directly affected by stretch-induced alterations in molecular structure (82). In any or all of these ways, mechanical forces can be incorporated into models of biomolecular signaling networks by accounting for these effects on biochemical and biophysical interaction parameters.

Importantly, physiochemical signaling is not constrained to one-way transformation of mechanical signals into biochemical ones. The reverse conversion also occurs and is most evident in the role of motor proteins. Chemical signals activate motor proteins that generate contractile forces along cytoskeletal elements such as actin filaments. These forces are then distributed onto filament termination sites such as cell-substratum contact where the force is imparted onto the local ECM proteins. This may mediate ECM remodeling and may also stretch ECM proteins to expose cryptic sites on these multidomain structures (83). In this way, interchange between chemical and mechanical signals relays commands from within the cell to the extracellular milieu through physiochemical "inside-out" signaling.

\section{TOWARD INTEGRATED NETWORK MODELS}

Alongside analysis of both biochemical and biophysical elements governing specific signaling pathways, it will be equally essential to integrate these pathways into a network model of signal transduction. In fact, models incorporating multiple pathways into a network structure reveal features not observed from analysis of individual pathways. Such emergent properties include self-sustaining signals resulting from positive feedback interactions between two pathways despite a transient stimulus, provided the stimulus passes a threshold level of magnitude and duration (84). 
Considering network models involving more interconnected pathways and including biophysical effects dramatically elevates the number of parameters and components that would be required to describe the hundreds of interactions in a one-level, mass-action kinetic model (85). A potentially effective and efficient approach we might propose, which could generate useful conceptual insights, would be to group signaling events into modules containing interrelated pathways with spatial or biochemical ties, enabling a two-level hierarchical approach wherein the overall signaling network is modeled in terms of process modules, and each module in terms of its underlying biochemical/biophysical molecular interactions.

Signaling modules would be defined as discrete units whose underlying mechanisms can be studied first in isolation and then integrated into a larger flow diagram of networked modules. One example module may be focal adhesion complexes composed of physically associated signaling and cytoskeletal proteins (86). This "solid-state" module receives several inputs, processes them through biochemical and biophysical interactions of constituent molecules, and yields multiple outputs (47). Efforts to analyze focal adhesion signal processing would provide this module's salient performance characteristics or rules of operation, which can then be incorporated into a network of modules without including all the mass-action kinetic equations for focal adhesion signal processing.

Alternatively, modules may be biochemical stand-alone units without requirement for spatial localization. One such closely studied module is the MAPK module of protein kinases, typically involving sequential activation of three kinases in an enzyme cascade (87) (Figure 1). Here, multiple events may collectively determine the activity of the first enzyme, after which the module operates based on intrinsic properties and yields an output-the activity level of the final kinase. Kinetic models for this module fully describing its operation would provide simple performance equations for this unit, which would allow easier integration of this module into a larger web of similarly defined process units.

Now, given the first level of well-characterized modules and their associated performance equations, what form is most appropriate for the second-tier overall network model that integrates these modules? Enzymatic reaction networks have been shown to emulate either Boolean or fuzzy logic functions such as AND, OR, and XOR, suggesting that some modules can best be represented in a computation algorithm framework (88). Alternatively, one could envision multimolecular assemblies serving process functions of various types, which can then be interconnected in different ways to yield diverse input/output functions; rough analogies to electrical circuit modules such as amplifiers might usefully stimulate modeling approaches. Combining such circuit representation of transcriptional regulation with biochemical kinetic descriptions has yielded successful simulation of $\lambda$ phage lysis-lysogeny decision $(89,90)$. Hence, in addition to incorporation into signaling circuits, modules may be networked in a manner similar to the assembly of unit operations into overall processes. Here, signaling outputs would be directed between different modules providing the interconnectivity, and optimization and network performance can be assessed from a process systems perspective. As frameworks 
are developed to model networks, it is likely that some hybrid of electrical circuit and process systems analysis will be necessary to integrate the biochemical and biophysical processes governing signal transduction and to estimate its performance over broad time and space scales.

\section{CONCLUDING REMARKS}

With the exciting likelihood that the Human Genome Project will consummate the complete DNA sequencing of the human genome, and high-throughput genomics and proteomics methods will reveal gene and protein expression profiles, there is an equally significant task of determining the functional role of these molecules, not in isolation but within a network of interactions. However, consideration of the interconnected biochemistry alone is not sufficient, as biophysical aspects provide an important layer of regulation, requiring novel imaging techniques for dynamically tracking spatial distributions of signaling proteins. Because these reactions and biophysical elements do not arrange into parallel, independent pathways but into an interconnected web, it hampers interpretation of experiments aimed at assigning function to newly identified proteins, as observations may not fit intuitive expectations of simple linear cause-effect relationships (91). Thus, prescribing function to signaling moieties likely must occur in parallel with predicting behavior of complex, integrated signaling networks through mathematical models and engineering analysis and synthesis.

\section{ACKNOWLEDGMENTS}

We thank Adam Arkin for helpful suggestions and valuable critique of our manuscript. This work was funded by the NIH Biotechnology Training Grant at MIT and an Anna Fuller Graduate Fellowship in Molecular Oncology from MIT Cancer Research Center to ARA, and NIH grant NIGMS 53905 and NSF grant BES 9727146 to DAL.

\section{Visit the Annual Reviews home page at www.AnnualReviews.org}

\section{LITERATURE CITED}

1. Miller A, Hafler DA, Weiner HL. 1991. Immunotherapy in autoimmune diseases. Curr. Opin. Immunol. 3:936-40

2. Watschinger B. 1995. How T cells recognize alloantigen: evidence for two pathways for allorejection. Nephrol. Dial. Transplant 10:1556-58

3. Pimentel E. 1994. Insulin. In Handbook of
Growth Factors: Peptide Growth Factors, 2:1-54. CRC Press. 362 pp.

4. Juliano R. 1994. Signal transduction by integrins and its role in regulation of tumor growth. Cancer Metastasis Rev. 13:25-30

5. Levitzki A. 1996. Targeting signal transduction for disease therapy. Curr. Opin. Cell. Biol. 8:239-44 
6. Hunter T. 1994. 1001 protein kinases redux-towards 2000. Semin. Cell Biol. 5: $367-76$

7. Hunter T. 1995. Protein kinases and phosphatases: the yin and yang of protein phosphorylation and signaling. Cell 80:225-36

8. van der Geer P, Hunter T. 1994. Receptor protein-tyrosine kinases and their signal transduction pathways. Annu. Rev. Cell. Biol. 10:251-337

9. Hanks SK, Polte TR. 1997. Signaling through focal adhesion kinase. Bioessays 19:137-45

10. Schwartz MA, Schaller MD, Ginsberg MH. 1995. Integrins: emerging paradigms of signal transduction. Annu. Rev. Cell Dev. Biol. 11:549-99

11. Clark EA, Brugge JS. 1995. Integrins and signal transduction pathways: the road taken. Science 268:233-39

12. Kholodenko BN, Hoek JB, Westerhoff HV, Brown GC. 1997. Quantification of information transfer via cellular signal transduction pathways. FEBS Lett. 414:430-34

13. Stephanopoulos GN, Aristidou AA, Nielsen J. 1998. Metabolic Engineering: Principles and Methodologies. San Diego: Academic. $725 \mathrm{pp}$.

14. Brown GC, Hoek JB, Kholodenko BN. 1997. Why do protein kinase cascades have more than one level? Trends Biochem. Sci. 22: 288

15. Goldbeter A, Koshland DE Jr. 1981. An amplified sensitivity arising from covalent modification in biological systems. Proc. Natl. Acad. Sci. USA 78:6840-44

16. Koshland DE Jr, Nemethy G, Filmer D. 1966. Comparison of experimental binding data and theoretical models in proteins containing subunits. Biochemistry 5:36585

17. Ferrell JE Jr. 1997. How responses get more switch-like as you move down a protein kinase cascade. Trends Biochem. Sci. 22:288-89

18. Huang C-YF, Ferrell JE. 1996. Ultrasensitivity in the mitogen-activated protein ki- nase cascade. Proc. Natl. Acad. Sci. USA 93:10078-83

19. Ferrell JE Jr. 1996. Tripping the switch fantastic: how a protein kinase cascade can convert graded inputs into switch-like outputs. Trends Biochem. Sci. 21:460-66

20. Ferrell JE Jr, Bhatt RR. 1997. Mechanistic studies of the dual phosphorylation of mitogen-activated protein kinase. J. Biol. Chem. 272:19008-16

21. DiMilla PA, Barbee K, Lauffenburger DA. 1991. Mathematical model for the effects of adhesion and mechanics on cell migration speed. Biophys. J. 60:15-37

22. Gurdon JB, Dyson S, Johnston D. 1998. Cells' perception of position in a concentration gradient. Cell 95:159-62

23. Dyson S, Gurdon JB. 1998. The interpretation of position in a morphogen gradient as revealed by occupancy of activin receptors. Cell 93:557-68

24. McAdams HH, Arkin A. 1997. Stochastic mechanisms in gene expression. Proc. Natl. Acad. Sci. USA 94:814-19

25. Arkin A, Ross J, McAdams HH. 1998. Stochastic kinetic analysis of developmental pathway bifurcation in phage $\lambda$-infected Escherichia coli cells. Genetics 149:163348

26. Tranquillo RT, Lauffenburger DA. 1987. Stochastic model of leukocyte chemosensory movement. J. Math. Biol. 25:229-62

27. Tranquillo RT, Lauffenburger DA, Zigmond SH. 1988. A stochastic model for leukocyte random motility and chemotaxis based on receptor binding fluctuations. $J$. Cell. Biol. 106:303-9

28. Zigmond SH. 1977. Ability of polymorphonuclear leukocytes to orient in gradients of chemotactic factors. J. Cell Biol. 75:606-16

29. Koshland DE Jr, Goldbeter A, Stock JB. 1982. Amplification and adaptation in regulatory and sensory systems. Science 217:220-25

30. Parkinson JS. 1993. Signal transduction schemes of bacteria. Cell 73:857-71 
31. Falke JJ, Bass RB, Butler SL, Chervitz SA, Danielson MA. 1997. The two-component signaling pathway of bacterial chemotaxis: a molecular view of signal transduction by receptors, kinases, and adaptation enzymes. Annu. Rev. Cell Dev. Biol. 13:457512

32. Spiro PA, Parkinson JS, Othmer HG. 1997. A model of excitation and adaptation in bacterial chemotaxis. Proc. Natl. Acad. Sci. USA 94:7263-68

33. Bourret RB, Borkovich KA, Simon MI. 1991. Signal transduction pathways involving protein phosphorylation in prokaryotes. Annu. Rev. Biochem. 60:401-41

34. Marshall CJ. 1995. Specificity of receptor tyrsosine kinase signaling: transient versus sustained extracellular signal-regulated kinase activation. Cell 80:179-85

35. Bottazzi ME, Zhu X, Bohmer RM, Assoian RK. 1999. Regulation of p21(cip1) expression by growth factors and the extracellular matrix reveals a role for transient ERK activity in G1 phase. J. Cell Biol. 146:125564

36. Deleted in press

37. Tombes RM, Auer KL, Mikkelsen R, Valerie K, Wymann MP, et al. 1998. The mitogen-activated protein (MAP) kinase cascade can either stimulate or inhibit DNA synthesis in primary cultures of rat hepatocytes depending upon whether its activation is acute/phasic or chronic. Biochem. J. 330:1451-60

38. Asthagiri AR, Nelson CM, Horwitz AF, Lauffenburger DA. 1999. Quantitative relationship among integrin-ligand binding, adhesion, and signaling via focal adhesion kinase and extracellular signal-regulated kinase 2. J. Biol. Chem. 274:27119-27

39. Thomas AP, Bird GS, Hajnoczky G, RobbGaspers LD, Putney JW Jr. 1996. Spatial and temporal aspects of cellular calcium signaling. FASEB J. 10:1505-17

40. Rooney TA, Sass EJ, Thomas AP. 1989. Characterization of cytosolic calcium oscillations induced by phenylephrine and vasopressin in single fura-2-loaded hepatocytes. J. Biol. Chem. 264:17131-41

41. Goldbeter A, Dupont G, Berridge J. 1990. Minimal model for signal-induced $\mathrm{Ca}^{2+}$ oscillations and for their frequency encoding through protein phosphorylation. Proc. Natl. Acad. Sci. USA 87:1461-65

42. Dolmetsch RE, Xu K, Lewis RS. 1998. Calcium oscillations increase the efficiency and specificity of gene expression. Nature 392:933-36

43. Oancea E, Meyer T. 1998. Protein kinase $\mathrm{C}$ as a molecular machine for decoding calcium and diacylglycerol signals.Cell 95:307-18

44. Meyer T. 1991. Cell signaling by second messenger waves. Cell 64:675-78

45. Tyson JJ. 1991. Modeling the cell division cycle: cdc2 and cyclin interactions. Proc. Natl. Acad. Sci. USA 88:7328-32

46. Koffas M, Roberge C, Lee K, Stephanopoulos G. 1999. Metabolic engineering. Annu. Rev. Biomed. Eng. 1:535-57

47. Bray D. 1998. Signaling complexes: biophysical constraints on intracellular communication. Annu. Rev. Biophys. Biomol. Struct. 27:59-75

48. Phillips RJ, Deen WM, Brady JF. 1989. Am. Inst. Chem. Eng. J. 35:1761-69

49. Dowd CJ, Cooney CL, Nugent MA. 1999. Heparan sulfate mediates bFGF transport through basement membrane by diffusion with rapid reversible binding. J. Biol. Chem. 274:5236-44

50. Flaumenhaft R, Moscatelli D, Rifkin DB. 1990. Heparin and heparan sulfate increase the radius of diffusion and action of basic fibroblast growth factor. J. Cell Biol. 111:1651-59

51. Gumbiner BM. 1998. Propagation and localization of Wnt signaling. Curr. Opin. Gen. Dev. 8:430-35

52. Bejsovec A, Wieschaus E. 1995. Signaling activities of the Drosophila wingless gene are separately mutable and appear to be transduced at the cell surface. Genetics 139:309-20 
53. Hays R, Gibori GB, Bejsovec A. 1997. Wingless signaling generates pattern through two distinct mechanisms. Development 124:3727-36

54. Sporn MB, Roberts AB. 1992. Autocrine secretion-10 years later. Ann. Intern. Med. 117:408-14

55. Lauffenburger DA, Oehrtman GT, Walker L, Wiley HS. 1998. Real-time quantitative measurement of autocrine ligand binding indicates that autocrine loops are spatially localized. Proc. Natl. Acad. Sci. USA 95:15368-73

56. Oehrtman GT, Wiley HS, Lauffenburger DA. 1998. Escape of autocrine ligands into extracellular medium: experimental test of theoretical model predictions. Biotechnol. Bioeng. 57:571-82

57. Neer EJ. 1995. Heterotrimeric G proteins: organizers of transmembrane signals. Cell 80:249-57

58. Shea LD, Omann GM, Linderman JJ. 1997. Calculation of diffusion-limited kinetics for the reactions in collision coupling and receptor cross-linking. Biophys. J. 73:2949-59

59. Faux MC, Scott JD. 1996. More on target with protein phosphorylation: conferring specificity by location. Trends Biochem. Sci. 21:312-15

60. Haugh JM, Lauffenburger DA. 1998. Analysis of receptor internalization as a mechanism for modulating signal transduction. J. Theor. Biol. 195:187-218

61. Haugh J, Schooler K, Wells A, Wiley H, Lauffenburger D. 1999. Effect of epidermal growth factor receptor internalization on regulation of the phospholipase C-gamma1 signaling pathway. J. Biol. Chem. 26:895865

62. Ferrell JE Jr. 1998. How regulated protein translocation can produce switch-like responses. Trends Biochem. Sci. 23:461-65

63. Schillace RV, Scott JD. 1999. Organization of kinases, phosphatases, and receptor signaling complexes. J. Clin. Invest. 103:76165
64. Pawson T, Scott JD. 1997. Signaling through scaffold, anchoring, and adaptor proteins. Science 278:2075-80

65. Lee DA, Noguchi T, Knight MM, O'Donnell L, Bentley G, Bader DL. 1998. Response of chondrocyte subpopulations cultured within unloaded and loaded agarose. J. Orthop. Res. 16:726-33

66. Stockwell RA. 1991. Cartilage failure in osteoarthritis: relevance of normal structure and function. A review. Clin. Anat. 4: 161-91

67. Urban JP. 1994. The chondrocyte: a cell under pressure. Br. J. Rheumatol. 33:9018

68. Sadoshima J, Izumo S. 1997. The cellular and molecular response of cardiac myocytes to mechanical stress. Annu. Rev. Physiol. 59:551-71

69. Sadoshima J, Jahn L, Takashashi T, Kulik TJ, Izumo S. 1992. Molecular characterization of the stretch-induced adaptation of cultured cardiac cells: an in vitro model of load-induced cardiac hypertophy. J. Biol. Chem. 267:10551-60

70. Vandenburgh HH, Kaufman S. 1979. In vitro model for stretch-induced hypertophy of skeletal muscle. Science 203:265-68

71. Davies PF, Barbee KA, Volin MV, Robotewskyj A, Chen J, et al. 1997. Spatial relationships in early signaling events of flow-mediated endothelial mechanotransduction. Annu. Rev. Physiol. 59:527-49

72. Papadaki M, Eskin SG. 1997. Effects of fluid shear stress on gene regulation of vascular cells. Biotechnol. Prog. 13:209-21

73. Wang D, Tarbell J. 1995. Modeling intersititial flow in an artery wall allows estimation of wall shear stress on smooth muscle cells. J. Biomech. Eng. 117:358-63

74. Ingber DE. 1997. Tensegrity: the architectural basis of cellular mechanotransduction. Annu. Rev. Physiol. 59:575-99

75. Shyy JY-J, Chien S. 1997. Role of integrins in cellular responses to mechanical stress and adhesion. Curr. Opin. Cell. Biol. 9:707-13 
76. Maniotis AJ, Chen CS, Ingber DE. 1997. Demonstration of mechanical connections between integrins, cytoskeletal filaments, and nucleoplasm that stabilize nuclear structure. Proc. Natl. Acad. Sci. USA 94:849-54

77. Galbraith CG, Sheetz MP. 1998. Forces on adhesive contacts affect cell function. Curr. Opin. Cell. Biol. 10:566-71

78. Chen K-D, Li Y-S, Kim M, Li S, Yuan $\mathrm{S}$, et al. 1999. Mechanotransduction in response to shear stress. J. Biol. Chem. 274:18393-400

79. Ohno M, Cooke JP, Dzau VJ, Gibbons GH. 1995. Fluid shear stress induces transforming growth factor beta-1 transcription and production: modulation by potassium channel blockage. J. Clin. Invest. 95:136369

80. Bell GI. 1978. Models for the specific adhesion of cells to cells. Science 200:61827

81. Khan S, Sheetz MP. 1997. Force effects on biochemical kinetics. Annu. Rev. Biochem. 66:785-805

82. Sokabe M, Sachs F. 1992. Towards molecular mechanism of activation in mechanosensitive ion channels. In Advances in Comparative and Environmental Physiology, ed. F Ito, 10:55-77. Berlin/New York: Springer-Verlag. 309 pp.

83. Hynes RO. 1999. The dynamic dialogue between cells and matrices: implications of fibronectin's elasticity. Proc. Natl. Acad. Sci. USA 96:2588-90

84. Bhalla US, Iyengar R. 1999. Emergent properties of networks of biological signaling pathways. Science 283:381-87

85. Weng G, Bhalla US, Iyengar R. 1999. Complexity in biological signaling systems. Science 284:92-96

86. Jockusch BM, Bubeck P, et al. 1995. The molecular architecture of focal adhesions. Annu. Rev. Cell. Dev. Biol. 11:379-416

87. Lewis TS, Shapiro PS, Ahn NG. 1998. Signal transduction through MAP kinase cascades. Adv. Cancer Res. 74:49-139
88. Arkin A, Ross J. 1994. Computational functions in biochemical reaction networks. Biophys. J. 67:560-78

89. McAdams HH, Shapiro L. 1995. Circuit simulation of genetic networks. Science 269:650-56

90. McAdams HH, Arkin A. 1998. Simulation of prokaryotic genetic circuits. Annu. Rev. Biophys. Biomol. Struct. 27:199-224

91. Brugge JS, McCormick F. 1999. Intracellular networking. Curr. Opin. Cell. Biol. 11:173-76

92. Rosales C, O'Brien V, Kornberg L, Juliano R. 1995. Signal transduction by cell adhesion receptors. Biochim. Biophys. Acta 1242:77-98

93. Woods A, Couchman JR. 1998. Syndecans: synergistic activators of cell adhesion. Trends Cell Biol. 8:189-92

94. Hynes RO. 1992. Integrins: versatility, modulation, and signaling in cell adhesion. Cell 69:11-25

95. Stappert J, Kemler R. 1999. The cadherin superfamily. Adv. Mol. Cell Biol. 28:2763

96. Crockett-Torabi E. 1998. Selectins and mechanisms of signal transduction. $J$. Leukocyte Biol. 63:1-14

97. Pawson T. 1994. SH2 and SH3 domains in signal transduction. Adv. Cancer Res. 64:87-110

98. Barinaga M. 1999. New clues to how proteins link up to run the cell. Science 283:1247-49

99. Lu P-J, Zhou XZ, Shen M, Lu KP. 1999. Function of WW domains as phosphoserine- or phosphothreonine-binding modules. Science 283:1325-28

100. Fanning AS, Anderson JM. 1999. PDZ domains: fundamental building blocks in the organization of protein complexes at the plasma membrane. J. Clin. Invest. 103: 767-72

101. Irvine R. 1998. Inositol phospholipids: translocation, translocation, translocation. .. Curr. Biol. 8:R557-59

102. Byon JC, Kenner KA, Kusari AB, Kusari 
J. 1997. Regulation of growth factorinduced signaling by protein-tyrosinephosphatases. Proc. Soc. Exp. Biol. Med. 216:1-20

103. Millward TA, Zolnierowicz S, Hemmings BA. 1999. Regulation of protein kinase cascades by protein phosphatase $2 \mathrm{~A}$. Trends Biochem. Sci. 24:186-91

104. Maehama T, Dixon JE. 1999. PTEN: a tumor suppressor that functions as a phospholipid phosphatase. Trends Cell. Biol. 9:125-28 


\section{CONTENTS}

PHYSICOCHEMICAL FOUNDATIONS AND STRUCTURAL DESIGN OF HYDROGELS IN MEDICINE AND BIOLOGY, $N$. A. Peppas, Y. Huang, M. Torres-Lugo, J. H. Ward, J. Zhang

BIOENGINEERING MODELS OF CELL SIGNALING, Anand $R$. Asthagiri, Douglas A. Lauffenburger

FUNDAMENTALS OF IMPACT BIOMECHANICS: Part I -

Biomechanics of the Head, Neck, and Thorax, Albert I. King

INJURY AND REPAIR OF LIGAMENTS AND TENDONS, Savio L.-Y. Woo, Richard E. Debski, Jennifer Zeminski, Steven D. Abramowitch, Serena S. Chan Saw, MS, James A. Fenwick

ELECTROPHYSIOLOGICAL MODELING OF CARDIAC VENTRICULAR FUNCTION: From Cell to Organ, R. L. Winslow, D. F. Scollan, A. Holmes, C. K. Yung, J. Zhang, M. S. Jafri

CRYOSURGERY, Boris Rubinsky

CELL MECHANICS: Mechanical Response, Cell Adhesion, and Molecular Deformation, Cheng Zhu, Gang Bao, Ning Wang

MICROENGINEERING OF CELLULAR INTERACTIONS, Albert

Folch, Mehmet Toner

QUANTITATIVE MEASUREMENT AND PREDICTION OF BIOPHYSICAL RESPONSE DURING FREEZING IN TISSUES, John

C. Bischof

MICROFABRICATED MICRONEEDLES FOR GENE AND DRUG DELIVERY, Devin V. McAllister, Mark G. Allen, Mark R. Prausnitz

CURRENT METHODS IN MEDICAL IMAGE SEGMENTATION, Dzung L. Pham, Chenyang Xu, Jerry L. Prince

NEW CURRENTS IN ELECTRICAL STIMULATION OF EXCITABLE

TWO-PHOTON EXCITATION FLUORESCENCE MICROSCOPY, Peter T. C. So, Chen Y. Dong, Barry R. Masters, Keith M. Berland IMAGING THREE-DIMENSIONAL CARDIAC FUNCTION, $W$. $G$. O'Dell, A. D. McCulloch

THREE-DIMENSIONAL ULTRASOUND IMAGING, Aaron Fenster, Donal B. Downey

BIOPHYSICAL INJURY MECHANISMS IN ELECTRICAL SHOCK

TRAUMA, Raphael C. Lee, Dajun Zhang, Jurgen Hannig

WAVELETS IN TEMPORAL AND SPATIAL PROCESSING OF

BIOMEDICAL IMAGES, Andrew F. Laine 
MICRODEVICES IN MEDICINE, Dennis L. Polla, Arthur G. Erdman, William P. Robbins, David T. Markus, Jorge Diaz-Diaz, Raed Rizq,

Yunwoo Nam, Hui Tao Brickner, Amy Wang, Peter Krulevitch

NEUROENGINEERING MODELS OF BRAIN DISEASE, Leif $H$.

Finkel

EXTRACORPOREAL TISSUE ENGINEERED LIVER-ASSIST

DEVICES, Emmanouhl S. Tzanakakis, Donavon J. Hess, Timothy D.

Sielaff, Wei-Shou Hu

607

MAGNETIC RESONANCE STUDIES OF BRAIN FUNCTION AND

NEUROCHEMISTRY, Kâmil Ugurbil, Gregor Adriany, Peter Andersen, Wei Chen, Rolf Gruetter, Xiaoping Hu, Hellmut Merkle, Dae-Shik Kim,

Seong-Gi Kim, John Strupp, Xiao Hong Zhu, Seiji Ogawa

INTERVENTIONAL AND INTRAOPERATIVE MAGNETIC

RESONANCE IMAGING, J. Kettenbach, D. F. Kacher, S. K. Koskinen, Stuart G. Silverman, A. Nabavi, Dave Gering, Clare M. C. Tempany, $R$.

B. Schwartz, R. Kikinis, P. M. Black, F. A. Jolesz

CARTILAGE TISSUE REMODELING IN RESPONSE TO MECHANICAL FORCES, Alan J. Grodzinsky, Marc E. Levenston, Moonsoo Jin, Eliot H. Frank

IN VIVO NEAR-INFRARED SPECTROSCOPY, Peter Rolfe 